\title{
A Virtual Audience System for Enhancing Embodied Interaction Based on Conversational Activity
}

\author{
Yoshihiro Sejima ${ }^{1}$, Yutaka Ishii ${ }^{2}$, and Tomio Watanabe ${ }^{3}$ \\ ${ }^{1}$ Graduate School of Science and Engineering, Yamaguchi University, \\ 2-16-1 Tokiwadai, Ube, Yamaguchi, Japan \\ ${ }^{2}$ Information Science and Technology Center, Kobe University, \\ 1-1 Rokkodai, Nada, Kobe, Japan \\ ${ }^{3}$ Faculty of Computer Science and System Engineering, Okayama Prefectural University \\ 111, Kuboki, Soja, Okayama, Japan \\ sejima@yamaguchi-u.ac.jp, ishii@kobe-u.ac.jp, \\ watanabe@cse.oka-pu.ac.jp
}

\begin{abstract}
In this paper, we propose a model for estimating conversational activity based on the analysis of enhanced embodied interaction, and develop a virtual audience system. The proposed model is applied to a speech-driven embodied entrainment wall picture, which is a part of the virtual audience system, for promoting enhanced embodied interaction. This system generates activated movements based on the estimated value of conversational activity in enhanced interaction and provides a communication environment wherein embodied interaction is promoted by the virtual audience. The effectiveness of the system was demonstrated by means of sensory evaluations and behavioral analysis of 20 pairs of subjects involved in avatar-mediated communication.
\end{abstract}

Keywords: Human Interaction, Nonverbal Communication, Virtual Communication, Enhanced Interaction, Virtual Audience.

\section{Introduction}

Advances in information technology have enabled humans to communicate through computer-generated (CG) characters called avatars in virtual worlds such as Second Life and online games [1]. Many studies on the development of communication systems using avatars [2]-[4] and evaluation of the embodied conversational agent in such communication systems [5],[6] have been performed. However, it is difficult to enhance the embodied interaction in these systems and to create an environment where the talker can share the sense of unity because the characteristics that enhance such embodied interaction has not been introduced thus far. Therefore, the development of an embodied interaction support system that enhances embodied interaction and increases talker involvement is essential.

In a previous study, we developed a speech-driven embodied entrainment system called InterWall wherein interactive CG sunflowers act as listeners [7]. This system 
can support human interaction and communication by generating embodied entrained movements such as nodding and body movements based on the speech input of a talker. We confirmed the importance of providing a communication environment in which not only the avatars but also CG objects placed around the avatars are related to virtual communication.

In this study, in order to promote enhanced embodied interaction, we analyzed the enhanced interaction through avatars by using an embodied virtual communication system. Then, we proposed a model for estimating conversational activity based on the above-mentioned analysis and developed a virtual audience system in which the proposed model is applied to InterWall. This system uses the proposed model to generate not only embodied entrained movements but also activated movements. The effectiveness of the developed system is demonstrated by means of sensory evaluations and behavioral analysis in an avatar-mediated communication system.

\section{Analysis of Enhancing Embodied Interaction}

\subsection{Experimental Method}

Using the embodied virtual communication system "EVCOS," two subjects indulged in a free conversation assuming that embodied interaction was enhanced. Figure 1(a) shows a sample virtual communication scene between two CG characters called VirtualActors (VAs). The VA is an interactive avatar that represents the subject's interactive behavior such as nodding and gestures on the basis of the nonverbal information provided by him/her in virtual space [8].

The experimental process was as follows. First, the subjects used the system for a 3-min free conversation. Here, the displayed images and the voices of both talkers were captured to record the communication scene using avatars. Next, they watched the video using Windows Media Player and picked up the enhanced interaction scene. Finally, non-participating subjects also watched the video using the same method (Figure 1(b)). The subjects were four pairs of male students.



(a)

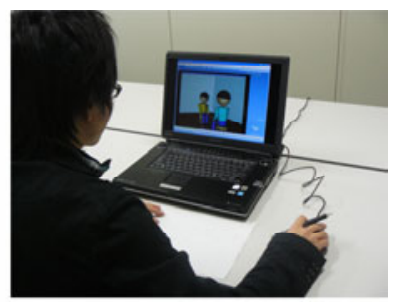

(b)

Fig. 1. Example of communication and picking-up scene 


\subsection{Analysis of Enhancing Interaction}

To detect the enhanced interaction period, we set the binary as the period of the communication scene selected by subjects as ON and the period otherwise as OFF. Figure 2 shows an example of the analysis results. The period indicated by the arrowed line was that of the communication scene selected by the subject. The same period is selected in spite of non-participating subjects.

A speech overlap was observed in live communication [9], [10]. Therefore, the enhanced interaction was analyzed by focusing on the speech overlap. Speech data $x(i)$ with a maximum amplitude of \pm 32767 was normalized at 1 and defined every 30 ms at a sampling rate of $11 \mathrm{kHz}$ as the binary burst-pause of speech generated with a fill-in value of $166 \mathrm{~ms}$. The overlap in the speech inputs of subjects 1 and 2 was evaluated by using the following expression.

$$
\begin{gathered}
\operatorname{overlap}(i)=x_{1}(i) \times x_{2}(i) \\
x_{1}(i): \operatorname{voice}(\text { talker1 }), \quad x_{2}(i): \text { voice }(\text { talker2) }
\end{gathered}
$$

A sample speech-overlap analysis result is shown in Figure 2. The figure shows that both the rate of speech overlap and degree of the histogram increase. Additionally, a positive correlation between the speech overlap and enhanced interaction is seen.

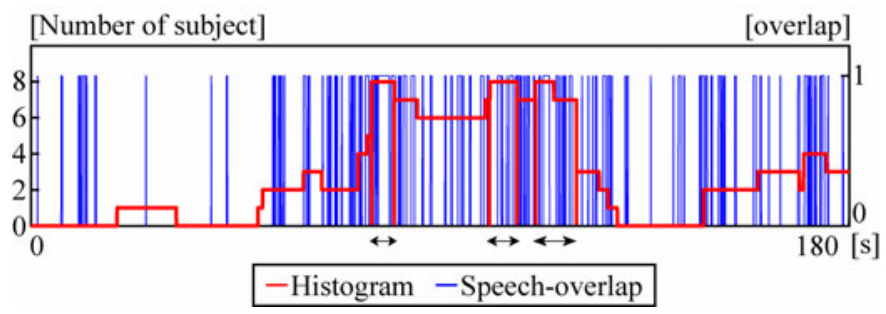

Fig. 2. Example of analysis of enhanced interaction

\section{Estimated Model of Conversational Activity}

Based on the analysis of the enhanced interaction, we propose a model for estimating the conversational activity from the degree of speech overlap. In this model, the present speech overlap is calculated from expression (1). Next, the conversational activity is estimated by introducing the calculated speech overlap to the weighted moving-average method from expression (2). The assignment of a weight to the present speech overlap causes the estimated value to increase rapidly. Figure 3 shows an example of the time changes in the estimated value and histogram. When the 
degree of the histogram increases, the estimated value also increases. Thus, the conversational activity can be estimated from the speech overlap.

$$
u(i)=\frac{\sum_{j=1}^{K}(K+1-j) \operatorname{overlap}(i-j)}{\sum_{j=1}^{K} j}
$$

$u(i)$ : conversational activity, $\quad \mathrm{K}:$ const.$(=150$ frame $)$

The enhanced interaction scene was preliminarily estimated on the basis of the established threshold from the estimated value using the model developed to estimate conversational activity. This threshold was set by a preliminary experiment. When the estimated value exceeded a threshold value, the period was estimated as the enhanced interaction scene.

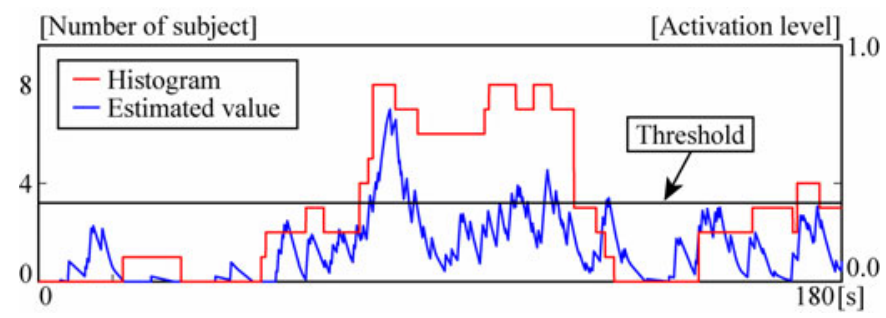

Fig. 3. Example of time changes in estimated value and histogram

\section{Virtual Audience System}

\subsection{Concept}

The concept of a virtual audience system is shown in Figure 4.

In human face-to-face communication, not only verbal messages but also nonverbal actions such as nodding and body movements are rhythmically related and mutually synchronized between the talkers [11]. This synchrony of embodied rhythms, called entrainment, in communication results in the sharing of embodiment in human interaction. By focusing on the entrainment in embodied communication, we have already analyzed the entrainment between the speech input of a speaker and nodding and body movements of a listener in face-to-face communication and have developed iRT (InterRobot Technology) that generates a variety of communicative actions and movements such as nodding and body movements on the basis of the speech input [12]. In addition, we have developed an interactive CG sunflowers called InterFlower and have demonstrated that InterFlower can effectively support human interaction and communication [13]. 
When the avatars are involved in an embodied interaction, the virtual audience generates interactive movements such as bending forward to enhance the embodied interaction; this leads to sharing of embodiment in virtual space and realizes smooth communication.

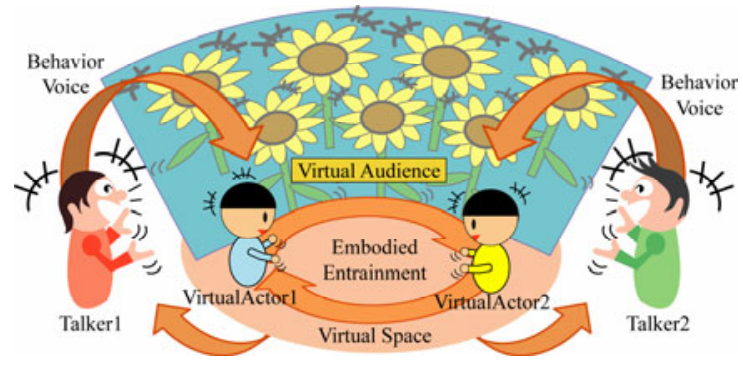

Fig. 4. Concept of virtual audience system

\subsection{System Setup}

Figure 5 shows the setup of the virtual audience system. The virtual space is generated using Microsoft DirectX 9.0 SDK on a workstation (HP Workstation Xw4200) running Windows XP. The movements of the head, arms, and body of each VA is measured in terms of their positions and angles using four magnetic sensors (Polhemus FASTRAK) placed on the subject's head, wrists, and back. The voice is sampled with 16 bits at $11 \mathrm{kHz}$. The movement and voice data of each VA are transmitted through Ethernet. The VAs are represented at a frame rate of $30 \mathrm{fps}$.

The virtual audience consists of textures of the same size as a wall in a virtual space. These textures are used to represent six CG sunflowers and the background. The sunflowers act as listeners by producing communicative actions and movements that are coherently related to the speech input [7]. When a subject's speech is inputted to the system, the CG sunflowers emulate nodding movements, and the leaves in these flowers emulate body movements. Nodding movement is defined as the falling-rising of the flower with respect to the vertical at a speed of $0.03 \mathrm{rad} / \mathrm{frame}$. Body movement is defined as the upward-downward motion of the leaf at a speed of 0.0125 $\mathrm{rad} /$ frame. In addition, when a value estimated using the proposed model exceeds the threshold value, the sunflowers exhibit enhanced movements such as bending forward. Bending-forward motion is defined as the falling-rising movement of the entire flower body with respect to the vertical at a speed of $0.012 \mathrm{rad} /$ frame. The sunflower leaves emulate enhanced movements defined as "wigwag" at a speed of $0.04 \mathrm{rad} /$ frame.

The moving-average (MA) model was used as the nodding response model [12]. The MA model estimates the nod timing $y(i)$ as the weighted sum of binary speech signal $x(i)$ in each frame of $1 / 30 \mathrm{~s}$, as shown in the following expression (3). 


$$
y(i)=\sum_{j=1}^{J} a(j) x(i-j)+w(i)
$$

$a(j)$ : linear prediction coefficient, $w(i):$ noise

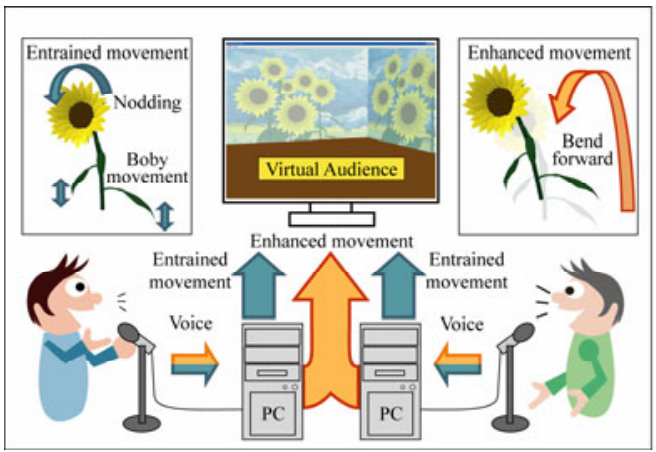

Fig. 5. Setup of virtual audience system

\section{Communication Experiment}

\subsection{Experimental Method}

The setup of the experiment in which two remote subjects converse from separate rooms is shown in Figure 6. The experiment was performed under the conditions of free conversation and consensus-building. In the free conversation experiment, two modes were compared: virtual-audience-generated entrained movements such as nodding and body movements (entrained mode) and virtual-audience-generated entrained and enhanced movements (combinational mode), because the effectiveness of entrained mode has been confirmed. In the consensus-building experiment, two modes were compared: the combinational mode and a static mode where the virtual audience does not move.

The experimental process was as follows. First, the subjects used the system to communicate with each other. Next, they used the system for a 3-min free conversation in each mode. Then, in each mode, they discussed specific topics without any time restriction. The topics discussed by the subjects were selected from the website "Goo Ranking," where ranking surveys are conducted on various topics, as listed in Table 1 [14]. In the consensus-building experiment, the subjects predicted the 1-3 rankings for the four highest-ranked items. They continued to converse until a consensus was arrived upon. Finally, they were instructed to perform a paired comparison of modes. In the paired comparison experiment, based on their preferences, they selected the better mode. 
The survey was conducted using a seven-point bipolar rating scale ranging from -3 (not at all) to 3 (extremely): 0 denotes the moderation after the completion of each mode. Each pair of subjects was presented with the two modes in a random order. Each pair of subjects belonged to the same sex (20 males and 20 females).

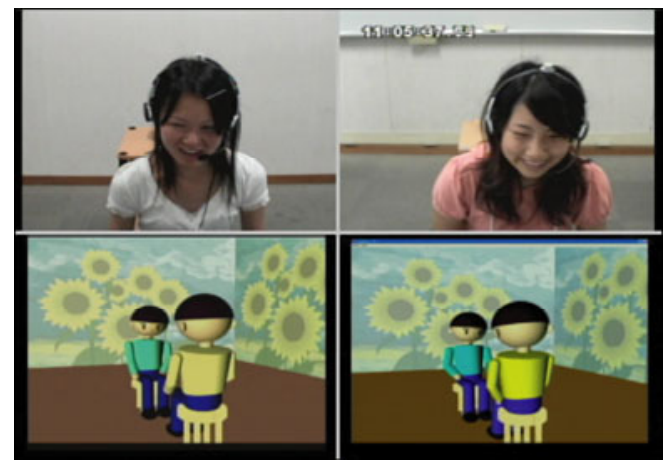

Fig. 6. Example scene of communication experiment

Table 1. Ranking of conversational topics discussed in the consensus-building experiment

\begin{tabular}{|c|c|}
\hline $\begin{array}{l}\text { The favorite competition } \\
\text { ranking of an athletic meet }\end{array}$ & $\begin{array}{l}\cdot \text { Tamaire } \\
\cdot \text { - Mock cavalry battle } \\
\cdot \text { Relay } \\
\cdot \text { Tug-of-war }\end{array}$ \\
\hline $\begin{array}{l}\text { The summer vacation's homework } \\
\text { in elementary school that remained } \\
\text { unfinished to the last moment }\end{array}$ & $\begin{array}{l}\cdot \text { Book report } \\
\text { - Independent research } \\
\cdot \text { Diary } \\
\cdot \text { Crafts }\end{array}$ \\
\hline
\end{tabular}

\subsection{Results of Sensory Evaluation}

The questionnaire results are shown in Figure 7. Figure 7 (1) shows the results of the sensory evaluation carried out in the free conversation experiment. From the results of the Wilcoxon signed-rank test, "enjoyment" and "excitement" were at the significant level of $1 \%$, and "preference" and "unification" were at 5\%. Figure 7 (2) shows the results of the sensory evaluation carried out in the consensus-building experiment. The significant level was set at $1 \%$ for all items. These results show the effectiveness of the system.

The results of the paired comparison are shown in Figure 8. In the free conversation experiment, the combinational mode was selected by $82.5 \%$ of the subjects (33 out of $40)$. In the consensus-building experiment, the combinational mode was selected by $92.5 \%$ of the subjects (37 out of 40 ). 


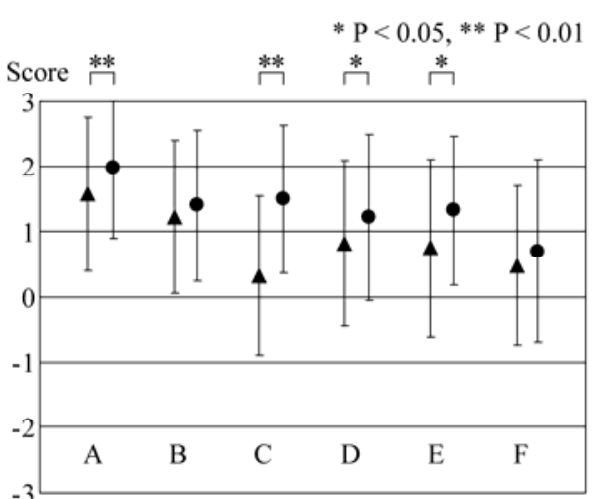

(1) Free conversation.

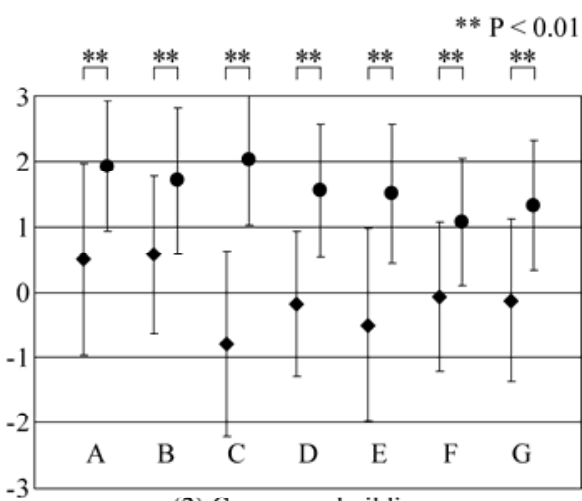

(2) Consensus building.

$\Delta$ entrained mode

- combinational mode

- static mode

Fig. 7. Result of sensory evaluation by questionnaire

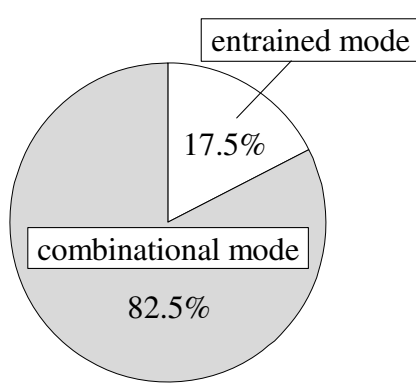

Free conversation

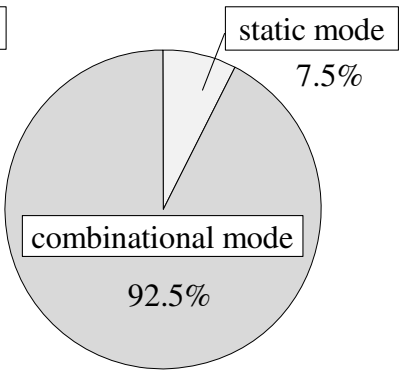

Consensus building

Fig. 8. Result of paired comparison

\subsection{Result of Behavioral Analysis}

The interaction among the subjects were analyzed by focusing on the conversational activity in the communication experiment. The rate of the estimated value of the proposed model was evaluated from expression (4) in each experiment.

$$
\text { Activity }=\frac{1}{N} \sum_{i=1}^{N} u(i)
$$

$u(i)$ : conversational activity, $N$ : conversational section

The analysis results are shown in Figure 9. The figure shows the rate of conversational activity based on the proposed model and the results of the statistical analysis. In the free conversation experiment, there was a significant difference of $1 \%$ 
between the combinational and entrained modes. In the consensus-building experiment, there was no significant difference between the two modes. These results show that embodied interaction is enhanced by a virtual audience in a free conversation.

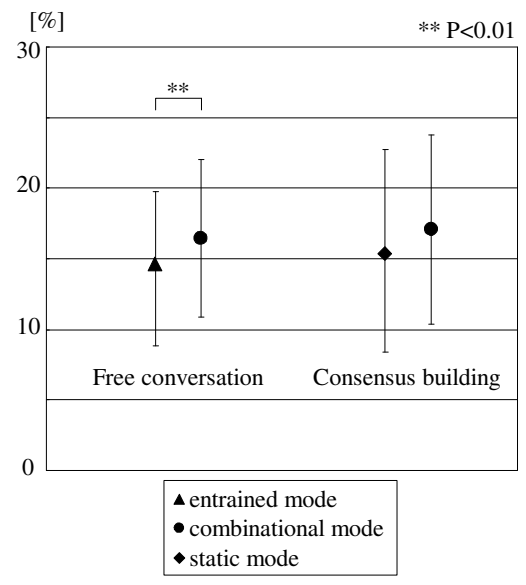

Fig. 9. Result of the rate of conversational activity

\section{Conclusion}

In this paper, we proposed a model to estimate conversational activity based on the speech overlap in conversation and developed a virtual audience system to enable enhanced embodied interaction. Using the system, we performed communication experiments and carried out a sensory evaluation and speech-overlap analysis under the conditions of free conversation and consensus-building. The results showed that the developed system effectively enhances embodied interaction.

Acknowledgments. This work was supported under the CREST (Core Research for Evolutional Science and Technology) of JST (Japan Science and Technology Agency).

\section{References}

1. Linden Lab, Second Life, http: / / secondli fe.com/

2. Miyajima, T., Fujita, K.: Control of avatar's facial expression using fundamental frequency in multi-user voice chat system. In: Proc. of the 6th International Conference on Intelligent Virtual Agents, p. 462 (2006)

3. Kusumi, T., Ogura, K., Miura, A.: The Development of a Positive Community using Virtual Space for Cancer Patients. In: Proc. of Second International Symposium on Universal Communication, pp. 490-493 (2008)

4. Kato, R., Yoshitomi, Y., Asada, T., Fujita, Y., Tabuse, M.: A Method for Synchronizing Nods of a CG Character and a Human Using Thermal Image Processing. In: Proc. of 18th IEEE International Symposium on Robot and Human Interactive Communication, pp. $848-853$ (2009) 
5. Michael, K., Patrick, G.: Igaze: Studying reactive gaze behavior in semi-immersive human-avatar interactions. In: Proc. of the 8th International Conference on Intelligent Virtual Agents, pp. 191-199 (2008)

6. Amy, L.B., Soyoung, K.: The Effects of Agent Nonverbal Communication on Procedural and Attitudinal Learning Outcomes. In: Proc. of the 8th International Conference on Intelligent Virtual Agents, pp. 208-214 (2008)

7. Sejima, Y., Watanabe, T.: A Speech-Driven Embodied Entrainment Wall Picture System for Supporting Virtual Communication. In: Proc. of 3rd International Universal Communication Symposium, pp. 309-314 (2009)

8. Watanabe, T., Ogikubo, M., Ishii, Y.: Visualization of respiration in the embodied virtual communication system and its evaluation. International Journal of Human-Computer Interaction 17, 89-102 (2004)

9. Ito, H., Shigeno, M., Nishimoto, T., Araki, M., Niimi, Y.: The Analysis of the Atmosphere in the Dialogs. Technical report of IPSJ, SLP, vol. 2002(10), pp. 103-108 (2002)

10. Nishimura, R., Kitaoka, N., Nakagawa, S.: Response Timing and Prosody Change Modeling in Conversations and Their Application to a Spoken Dialog System. In: Proc. Of the 48th SIG-SLUD of the Japanese Society for Artificial Intelligence, pp. 37-42 (2006)

11. Condon, W.S., Sander, L.W.: Neonate movement is synchronized with adult speech. Science 183, 99-101 (1974)

12. Watanabe, T., Okubo, M., Nakashige, M., Danbara, R.: Interactor: Speech-driven embodied interactive actor. International Journal of Human-Computer Interaction 17, 4360 (2004)

13. Yoshida, M., Watanabe, T., Yamamoto, M.: Development of a Speech-Driven Embodied Entrainment System with 3DCG Objects. Transactions of Human Interface Society 9(3), 87-96 (2007)

14. goo ranking (November 3, 2008), http: //ranking.goo.ne.jp/ 